\title{
Homeopathic Remedies Will Be the Pharmacological Means of Evidence for Informational Medicine
}

\author{
Mordeniz Cengiz* \\ Department of Anesthesiology, Intensive Care, and Pain Medicine, Medical Faculty of Namık Kemal University, Turkey
}

Submission: June 26, 2016; Published: July 14, 2017

*Corresponding author: Mordeniz Cengiz, Department of Anesthesiology, Intensive Care, and Pain Medicine, Medical Faculty of Namık Kemal University, Tekirdag, Turkey, Tel: +905055107675; Fax: 90282250 9950; Email: cengizmorster@gmail.com

\begin{abstract}
Homeopathy follows the rule of similar and uses potentated substances called remedies carrying information. Although some empirical evidence exists, there is little academic research and lack of a theoretical model to explain its effects. Homeopathy uses two instances of generalized entanglement: one between the remedy and the original substance (potentiation principle) and one between the individual symptoms of a patient and the general symptoms of a remedy picture (similarity principle). The remedy, itself is one entangled state and the homeopathic method is another entangled state between the symptomatology of the patient and the remedial substance. The homeopathic remedy is the substance which is not present and would become a kind of receptacle which 'absorbs' symptoms the whole symptom picture is also coherent.

Homeopathic treatment acts on the information level, but no physical explanation is given yet for the nature of the energy changes. Quantum mechanics seems to be a way to understand the success of homeopathic medicine in a physical context. To explain the therapeutic mode of homeopathic action, both 'local' bio-molecular mechanisms, such as the memory of water and 'non-local' macro-entanglement, such as patientpractitioner-remedy (PPR) can be imagined as interacting quantum-like fields; patients and practitioners in terms of quantum matter-type fields, and remedies and diseases as quantum interaction-type fields. Since quantum properties can be physical without being observable, a similarity could exist between homeopathy and quantum theory which could be useful for modeling the homeopathic process.
\end{abstract}

Keywords: Homeopathy; Quantum mechanics; Entanglement; Coherence; Remedy

Abbreviations: RCT : Randomized Controlled Trial; NPs: Nano Particles; PPR: Patient-Practitioner-Remedy

\section{Introduction}

Homeopathy is based on 3 premises: the law of similars; individualized therapy based on broadly defined symptoms; and the use of very small doses.The law of similars is the theory that a plant, animal, or mineral substance that causes a particular set of symptoms in a healthy person, when administered in a homeopathic dosage form to a sick person exhibiting those symptoms, will cure them. The practice of individualized therapy based on symptoms is grounded on the premise that every patient has a unique personality and symptom pattern of illness, meaning that the same biomedical disease manifests in subtly different ways in each person. Thus, each homeopathic remedy must be chosen by a homeopathic practitioner, so that it most closely addresses each unique variation. The use of very small doses is important because often the substances used to make the remedy are toxic at undiluted concentrations. The diluting process involves succussion, vigorous shaking of the original substance with alcohol or water, which activates and "potenizes" the remedy. Homeopathic products are often diluted so much that conventional pharmacology tells us that no molecules of the original solute remain in the finished product. The fundamental tenets of homeopathy are completely different from modern medicine, pharmacology, and chemistry. Main sources of contention include: the implausibility of homeopathic principles; the lack of a proven or plausible mechanism of action for homeopathy; and mixed results from randomized, controlled trials on homeopathic preparations [1].

Homeopathy is a system of alternative medicine created in 1796 by Samuel Hahnemann based on his doctrine of like cures like according to which a substance that causes the symptoms of a disease in healthy people will cure similar symptoms in sick people. The National Center for Complementary and Alternative Medicine of the United States 'National Institutes of Health states: 
Homeopathy is a controversial topic in complementary medicine research. A number of the key concepts of homeopathy are not consistent with fundamental concepts of chemistry and physics. For example, it is not possible to explain in scientific terms how a remedy containing little or no active ingredient can have any effect. This, in turn, creates major challenges to rigorous clinical investigation of homeopathic remedies. For example, one cannot confirm that an extremely dilute remedy contains what is listed on the label, or develop objective measures that show effects of extremely dilute remedies in the human body.

Nan particles (a few to a few thousand atoms) have properties different from those found in bulk (billions of atoms) forms of the "same" material. These altered properties include changes in electromagnetic, thermal, optical, biochemical, and quantum properties of the particle [2]. Homeopathy treats patient on the basis of totality of symptoms and is based on the principle of 'like cures like'. It uses ultra-low doses of highly diluted natural substances as remedies that originate from plants, minerals or animals [3].

Homeopathic preparations are used in homeopathy and anthroposophic medicine. Although there is evidence of effectiveness in several clinical studies, including doubleblinded randomized controlled trials, their nature and mode of action could not be explained with current scientific approaches yet. Several physical methods have already been applied to investigate homeopathic preparations but it is yet unclear which methods are best suited to identify characteristic physicochemical properties of homeopathic preparations [4]. The source material of homeopathic medicines is diluted past Avogadro's number of molecules. The nanoheterogenous structure of water can be determined by interactive phenomena such as epitaxy (the transmission of structural information from the surface of one material to another without the transfer of any matter), temperature-pressure processes during succussion, and formation of colloidal nanobubbles containing gaseous inclusions of oxygen, nitrogen, carbon dioxide, and possibly the remedy source material [5].

The individual symptom picture and the commonality of the remedy symptoms are the complementary descriptions that create the entangled state. The success of this entanglement depends, on the correctness of finding the similar remedy of the individual symptomatology. If this entanglement between remedy picture and individual symptomatology is created, then any symbol or sign representing the substance, which is in fact not present, will do the job.

\section{Discussion}

The Memory of Water, a "local” explanation of homeopathy's efficacy, envisages "quantum macro-entanglement" among patient, practitioner, and remedy to form a "PPR" entangled state, from which the possibility of cure may manifest. Semiotic analysis affords a geometrical description of this entangled state as a patient-centered chiral tetrahedron depicting three different types of symptoms (of the patient, the dis-ease, and the remedial substance) and the potentized remedy [6].

Quantum teleportation applications always use doubleentangled states and a weak channel of communication, to decode the original signal,.. In homeopathy, there is an analogous situation that traces the original substance as a classically conceived information channel as imprints. According to "Avogadro's constant", after a total of 24 " $\mathrm{x}$ " dilutions or 12 "c" dilutions, no particles of the original substance should theoretically be present in these preparations. At these small scales, the dynamics of the chemical machinery is governed by the laws of quantum mechanics. Higher dilution of homoeopathic medicines are no more placebo and the action of such medicine is due to quantum mechanics. From scientific evidence, it is shown that homoeopathic medicine in diluted form are no more placebo i.e. Shi et al showed that Calcium Phosphate Nanoparticles (NPs) Induce Apoptosis in Osteosarcoma Cells, Das et al showed that homeopathic Phytolacca tincture encapsulated as nanoparticles have chemopreventive effects in human lung adenocarcinoma cells [7].

In any pharmacologic treatment, the therapeutic effects have two aspects: specific effects (dosage, duration, means of application, Pharmacodynamics, pharmacokinetics, interaction between medications and others) and nonspecific effects (history and natural evolution of the disease, regression to the mean, socio environmental aspects, inter and intra-individual variability, yearning for healing, expectations and belief in the treatment, doctor-patient relationship, non-pharmacologic characteristics of the medication and others). The placebonocebo phenomenon is an important component with the doctor-patient relationship and the placebo response could be dominated by goals, desires, and expectations and associated emotions. The somatic perception of internal changes triggers positive feedback of these factors, selecting more positive emotional feeling to avoid aversive experiences, or to obtain desirable experiences.

An important placebo-inducing psychological mechanism is the conscious expectation of the patients of clinical improvement. Suggestions to which the patient is exposed (family members, friends, doctors etc.) may also alter the expectations concerning the treatment. In the absence of a control group (natural evolution, no treatment) other non-specific factors that could influence the therapeutic effect should also be investigated, such as seasonal changes, regression to the mean, the Hawthorne effect, beneficial effects of additional treatment.

Homeopathy, with its differentiated and humanized doctorpatient relationship with whole healing and free of adverse effects, positively influences patients' expectations. This may stimulate the activity of specific areas of the brain and liberation of specific neurotransmitters, causing non-specific therapeutic 
effects [8]. Classical homeopathy represents individuality related to the subjective experience of the patient. Two variables appear to be central aspects of the homeopathic therapy: 'meaning' in terms of the patient's identification with the therapy received and patient-practitioner agreement in terms of the patient's well respected subjective perspective. Since the nature of the homeopathic therapy relies on a double entanglement process, the remedy itself does not do anything by itself. It might well be the case that the consciousness of the practitioner and the patient likewise plays another important role [9]. Both the biological growth and the change in concentration of the solvent during potentization of the homeopathic medicine belong to the class of quasi-quantum phenomena [10].

The analysis of some implications of three major properties of complex systems, their non-linearity, their self-organization capability, and their dynamicity, may illuminate possible links between complexity theory and the principles of homeopathy. In fact, the starting point for understanding the action of homeopathic medicines is not just the medicine itself. The homeopathic medicine might act in the patient as an external guide frequency to regulate homeostasis in non-linear, nonequilibrium living systems. A potentized homeopathic drug might be regarded as a small amount of matter containing elements oscillating coherently and communicating with the oscillatory frequencies, via a process of resonance, to biological fluids (mainly water). There is a link between drug frequencies and oscillators present in the living organism perturbed by the disease. The core of pathological dynamics is a loss of communication, and healing is the increase of communication, establishment of connections between different systems, integration of responses. Sometimes, healing requires external perturbations that remove pathological adaptations.

The two main goals of homeopathic therapy are to increase network connectivity or complexity and-in chronic diseases- to shift the pathology towards the healthy state. The homeopathic approach introduces systemic regulation, to recruit the associative memory of network that is coherent with its endogenous healing capability. The medicine should carry a global pattern of information to increase network connectivity at the specific points where it was lost. A 'homeopathic' medicine increaes the network connectivity by introducing information perturbing its nodes in a pattern 'similar' to the disease.

The purpose of a randomized controlled clinical trial is to assess whether a therapy is associated more frequently with a positive outcome than a placebo or a reference treatment. Blind RCTs are an inadequate research tool for testing complex therapies such as homeopathy. The negative effect of blinding on the success of homeopathy trials and the 'smearing effect' ('specific' effects of homeopathy medicine occurring in the placebo group) are described by quantum-like probabilities. Besides the 'classical' hypothesis of local causality, other authors used concepts derived from quantum physics, such as non-locality and entanglement. The authors who apply quantum concepts to homeopathy differ in what is entangled among practitioner, patient, and medicine $[11,12]$. Instead of formulating quantum mechanics with the use of classical analogies, Conte has fixed quantum reality on three basic and unclassical features: integer quanta, the non-commutation and the intrinsic, irreducible indeterminism and quantum interference. Mind like entities modulate matter with cognition ab initio, matter no more may be conceived by itself, or independently from cognition. In this scheme, cognition does not remain an abstract entity but becomes a quantum observable as a factor of knowledge that goes in line with the dynamics of the matter in quantum reality [13].

\section{Conclusion}

Homeopathy deserves an open-minded opportunity to demonstrate its value by using evidence-based principles, A quantum interpretation of the homeopathic method has shown that provided neither the medication itself, nor the patient is observed, a net effect is expected, even at homeopathic dilutions. Quantum mechanics seems to be the only way to understand the success of homeopathic medicine in a physical context.

\section{References}

1. Johnson T, Boon H (2007) Where Does Homeopathy Fit in Pharmacy Practice? American Journal of Pharmaceutical Education 71(1).

2. Navneet Omprakash Soni (2014) Homoeopathy from Nano Medicine to Quantum Medicine. International Journal of Ayurvedic and Herbal Medicine 4(6): 1671-1677.

3. Anisur Rahman Khuda-Bukhsh and Surajit Pathak (2008) Homeopathic Drug Discovery; Theory Update And Methododlogical Aspect. ExperT Opinion On Drug Discovery 3(8): 979-990.

4. Ursula Wolf, Martin Wolf, Peter Heusser, André Thurneysen, Stephan Baumgartner (2011) "Homeopathic Preparations of Quartz, Sulfur and Copper Sulfate Assessed by UV-Spectroscopy". Evidence-Based Complementary and Alternative Medicine 2011: 1-11.

5. Manju Lata Rao, Rustum Roy, Iris R Bell, Richard Hoover (2007) The defining role of structure in the plausibility of homeopathy. Homeopathy 96(3): 175-182.

6. Lionel R Milgrom (2008) A New Geometrical Description of Entanglement and the Curative Homeopathic Process. The Journal of Alternative and Complementary Medicine14(3): 329-339.

7. Marcus Z Teixeira, Cristina H F F Guedes, Patrıcia V Barreto, Milton A Martins (2010) The Placebo Effect And Homeopathy. Homeopathy 99(2): 119-129.

8. RT Mathie (2003) The Research Evidence Base For Homeopathy: A Fresh Assessment Of The Literature. Homeopathy 92: 84-91.

9. Tobias Nuhn, Rainer Ludtke, Max Geraedts(2010) Placebo Effect Sizes In Homeopathic Compared Toconventional Drugs- A Systematic Review Of Randomised Controlled Trials. Homeopathy 99: 76-82.

10. Marcin Molski (2011) Quasi-quantum model of potentization. Homeopathy 100(4): 259-263.

11. P Bellavite (2003) Complexity Science And Homeopathy: A Synthetic Overview. Homeopathy 92: 203-212.

12. Francis Beauvais (2013) A Quantum-Like Model Of Homeopathy Clinical Trials: Importance Of İn Situ Randomization And Unblinding. Homeopathy 102(2): 106-113. 
13. Conte Elio (2013) A Clifford algebraic analysis gives mathematical explanation of quantization of quantum theory and delineates a model of quantum reality in which information, primitive cognition entties and a principle of existence are intrinsically represented ab initio. World journal of Neuroscience 3(3): 157-170.

\section{Your next submission with Juniper Publishers will reach you the below assets}

- Quality Editorial service

- Swift Peer Review

- Reprints availability

- E-prints Service

- Manuscript Podcast for convenient understanding

- Global attainment for your research

- Manuscript accessibility in different formats ( Pdf, E-pub, Full Text, Audio)

- Unceasing customer service

Track the below URL for one-step submission https://juniperpublishers.com/online-submission.php 